\title{
A Scoping Review of Patient Preferences for HIV Self-Testing Services in the United States: Implications for Harm Reduction
}

This article was published in the following Dove Press journal: Patient Preference and Adherence

\author{
Mary E Hawk $\mathbb{D}^{1-3}$ \\ Ashley Chung $\mathbb{1 D}^{4}$ \\ Stephanie L Creasy ${ }^{1-3}$ \\ James E Egan ${ }^{1-3}$
}

'Behavioral and Community Health Sciences, Graduate School of Public Health, University of Pittsburgh, Pittsburgh, PA, USA; ${ }^{2}$ Center for LGBT Health Research, Graduate School of Public Health, University of Pittsburgh, Pittsburgh, PA, USA; ${ }^{3}$ Evaluation Institute, Behavioral and Community Health Sciences, Graduate School of Public Health, University of Pittsburgh, Pittsburgh, PA, USA; ${ }^{4}$ Jewish Healthcare Foundation, Pittsburgh, PA, USA
Correspondence: Mary E Hawk Behavioral and Community Health Sciences, Graduate School of Public Health, University of Pittsburgh, I 30 DeSoto Street, Pittsburgh, PA I526I, USA

Tel + I-4I2-648-2342

Email mary.hawk@pitt.edu
Purpose: Despite marked progress in the ability to test for, treat, and prevent HIV, the epidemic remains a significant public health concern, especially among key populations including prisoners; sex workers; transgender individuals; gay, bisexual, and other men who have sex with men (MSM); and Black and Latinx MSM. This scoping review was conducted to provide an overview of the current research describing patient preferences for HIVST in the United States to understand what key populations value about HIVST and why they are willing to use it. A targeted search for published literature on patient preferences for HIVST was conducted using Ovid Medline, PsychINFO, and an HIVST research database. Results: We reviewed 700 abstracts and 139 full texts. We found 19 articles published between January 2014 and April 2020 that included findings related to HIVST preferences. Overall, HIVST was preferred to more traditional testing. Six primary factors emerged as important HIVST values including: 1) convenience, 2) type of test; 3) cost, 4) stigma reduction, 5) risk reduction, and 6) self-control. Linkage to care was also identified as a key factor when considering HIVST as an option. Much of what makes HIVST attractive to individuals is their ability to self-determine how HIVST can be best integrated into their lives as a harm reduction tool for stigma and sexual risk mitigation.

Conclusion: While there is substantial evidence suggesting HIVST is feasible for use and there are aspects of HIVST that are beneficial and preferred over traditional testing approaches, there is a lack of rigorous implementation studies exploring how best to scale up HIVST in community settings. HIVST has the potential to be a powerful biobehavioral HIV prevention and harm reduction tool to empower individuals to engage with testing on their own terms while providing pathways to prevention and care support.

Keywords: HIV, self-testing, HIVST, harm reduction, United States, patient preferences

\section{Introduction}

Despite marked progress in the ability to test for, treat, and prevent HIV, the epidemic remains a significant public health concern, especially among key populations including prisoners; sex workers; transgender individuals; gay, bisexual, and other men who have sex with men (MSM); and Black men who have sex with men (BMSM). ${ }^{1,2}$ There is ample evidence that populations overrepresented in the HIV epidemic are those who experience barriers to care and are less likely to be engaged in all stages of the HIV care and prevention continuum including diagnosis, linkage to and retention in care, antiretroviral use, viral suppression, and connection to preexposure prophylaxis (PrEP) and PrEP care resources. ${ }^{2-5}$ 
As the first step in the continuum of care, improving HIV testing rates is vital. Although detection and case finding are of primary importance in getting people linked to HIV care or biobehavioral prevention opportunities, testing rates among all key populations remain far below the United Nations target of 90-90-90..$^{2,6-10}$ One approach to improving HIV testing rates that may be critically important for vulnerable and oppressed populations is HIV self-testing (HIVST). HIVST was approved in the United States (US) in 2012 as an oral rapid HIV test for sale in stores and online. ${ }^{11}$ Although a fingerstick HIV self-test is also available over-the-counter, this test requires individuals to send dried blood spots to a lab to receive results and therefore is not a method of rapid HIVST. ${ }^{12}$ HIVST has been used or piloted in healthcare settings, ${ }^{13,14}$ emergency departments, ${ }^{15,16}$ pharmacies, ${ }^{17-19}$ and mobile- or street-based testing, ${ }^{20,21}$ but most frequently is discussed as a mechanism for individuals to test themselves or partners for HIV in their homes or other private settings. Despite early concerns that learning one's HIV status independent of healthcare providers might lead to psychological, social, or medical harm, there is little evidence to date that such harm occurs. ${ }^{22}$ HIVST is a reliable and accurate method of HIV rapid diagnosis that can be used effectively by multiple populations. ${ }^{14,23-25}$ Further, there is evidence suggesting this method can increase HIV testing uptake and early diagnosis. $^{26-33}$

HIVST is particularly relevant for stigmatized populations as it provides individuals with increased privacy and greater control over their testing needs. ${ }^{34}$ The option to test privately at times and locations of choice creates the opportunity to reduce stigma, a significant barrier for MSM, ${ }^{35-39}$ BMSM,,${ }^{36,39-42}$ sex workers, ${ }^{43,44}$ prisoners, ${ }^{45,46}$ and transgender women. ${ }^{37,39,47}$ There is also evidence that HIVST may be acceptable for partner-testing by $\mathrm{MSM}^{48}$ and transgender women ${ }^{49}$ including transgender female sex workers. ${ }^{50}$ Several systematic or literature reviews discuss conditions for HIVST use among key populations, including one demonstrating that individuals who self-initiate HIVST are those having perception of risk for HIV and knowledge about HIV testing availability. ${ }^{27} \mathrm{~A}$ recent literature review found high rates of acceptability of HIVST among MSM but notes that values and preferences of other key populations remain understudied. ${ }^{51}$

There are some concerns that HIVST is sub-optimal compared to testing in clinical settings, both in terms of test sensitivity when comparing oral versus blood-based results and options for linkage to care. ${ }^{23}$ However, given barriers to care experienced by key populations including stigma, lack of access, and outreach that does not fully meet the needs of vulnerable and oppressed key populations, HIVST is an important option for empowering potential users to learn their HIV status in ways most responsive to their needs. ${ }^{41,42}$ Additionally, HIVST can decrease harm to participants, either directly, such as by reducing potential for intimate partner violence, or indirectly, by reducing stigma. HIVST, therefore, is consistent with the harm reduction model of care because it (1) aims to improve health outcomes by decreasing harm but not necessarily eliminating it and (2) is patient-centered and prioritizes the need, context, and autonomy of the patient. $^{52,53}$

Despite the body of literature describing acceptability, reliability, and participant characteristics that increase likelihood of using HIVST, it is also important to understand what individuals like about HIVST and the degree to which they prefer it to other testing methods. This scoping review was conducted to provide an overview of the current research describing patient preferences for HIVST in the US, in order to understand what members of key populations value about HIVST and why they are willing to use it.

\section{Methods}

An initial search for published literature on patient preference for HIVST was conducted by a health sciences librarian on April 9, 2020, via two electronic databases: Ovid Medline and PsychINFO. Concepts informing the search were HIV self-testing, HIVST, home test, and selftest. A combination of Medical Subject Heading (MeSH) terms and title, abstract, and keywords were used to develop the Medline search, which was then adapted to PsycINFO (Table 1. Summary of Databases Searched, Table 2. Medline Search Strategy, Table 3. PsycINFO Search Strategy). Only articles in English were included. A subsequent search was performed on April 14, 2020, via www.HIVST.org, a collaborative online resource dedicated to HIV self-testing research and policy information. Only peer-reviewed published articles were included; conference abstracts and grey literature were excluded. Systematic or other types of reviews were excluded after citation mining for any source studies that met inclusion criteria. Duplicate records were removed, and the remaining studies were screened for inclusion via abstract review. 
Table I Summary of Databases Searched

\begin{tabular}{|l|l|l|l|l|}
\hline Table & $\begin{array}{l}\text { Vendorl } \\
\text { Interface }\end{array}$ & Database & $\begin{array}{l}\text { Date } \\
\text { Searched }\end{array}$ & $\begin{array}{l}\text { Database } \\
\text { Update }\end{array}$ \\
\hline 2 & Ovid & Medline & April 9, 2020 & $\begin{array}{l}\text { 1946 to April 8, } \\
2020\end{array}$ \\
3 & Ovid & Psyclnfo $^{\circledR}$ & April 9, 2020 & $\begin{array}{l}806 \text { to March } \\
\text { Week 5 2020 }\end{array}$ \\
\hline
\end{tabular}

Note: Data from Niederstadt and Droste. ${ }^{74}$

Table 2 Medline Search Strategy

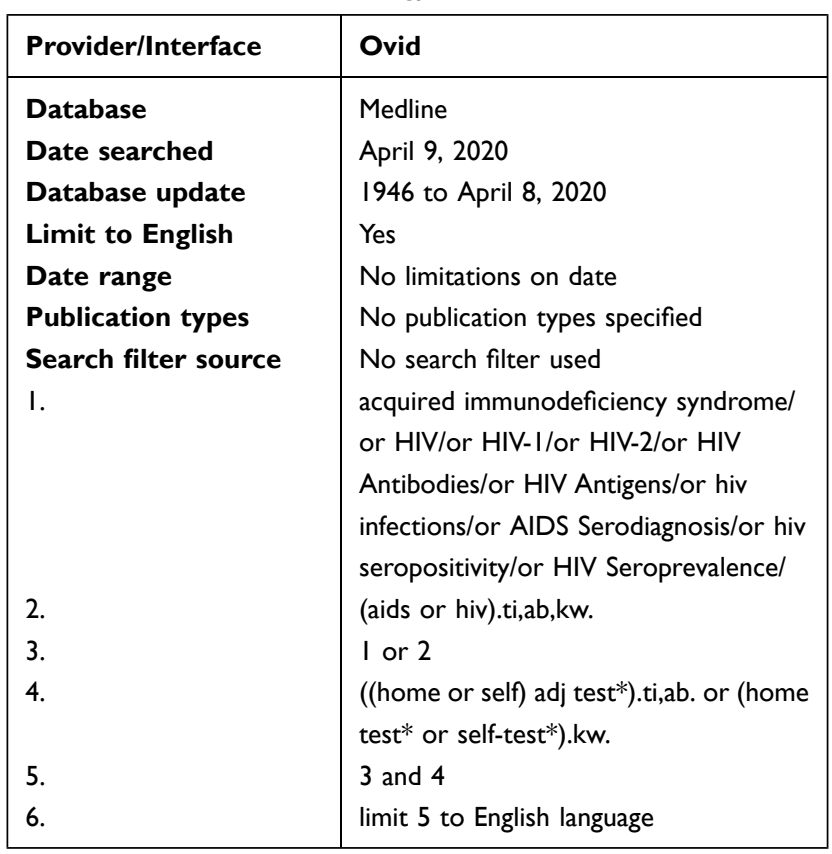

Table 3 PsycINFO ${ }^{\circledR}$ search strategy

\begin{tabular}{|l|l|}
\hline Provider/Interface & Ovid \\
\hline Database & Psyclnfo \\
Date searched & April 9, 2020 \\
Database update & I806 to March Week 5 2020 \\
Limit to English & Yes \\
Date Range & No limitations on date \\
Publication Types & No publication types specified \\
Search filter source & No search filter used \\
I. & hiv/or aids/or "aids (attitudes toward)"/ \\
& or aids prevention/ \\
2. & (aids or hiv).ti,ab,id. \\
3. & I or 2 \\
4. & (((self or home) adj test*) or HIVST).ti, \\
ab. or (home test* or self-test*).id. \\
5. & 3 and 4 \\
6. & limit 5 to English language \\
\hline
\end{tabular}

Studies that were advanced to full-text review met the following inclusion criteria: based in the US, published after 2012 following the FDA's approval of HIVST in the
United States, and having findings that describe patient preferences related to HIVST. Studies that did not address patient preferences for HIVST as a primary research question but did provide data regarding patient preferences were included. The research team discussed the meaning of "patient preferences" at length and ultimately focused its review on studies that explored why people preferred HIVST over other forms of testing, what participants valued about HIVST, and why people were willing to use HIVST. Studies that simply determined that people were willing to use HIVST without exploring reasons for their willingness were not included in the scoping review results. Similarly, studies that described participant characteristics or populations with increased likelihood of using HIVST without exploring what they valued about HIVST were excluded. Each study that was included in our review was read by at least two members of the study team to ensure data were accurately captured.

While searching for articles on testing patient preference, we noticed several terms that appeared repeatedly and often interchangeably. Here we include definitions of terms often used to describe patients' responses to HIVST in order to further clarify our decision-making process for including or excluding articles in our review.

\section{Acceptability}

Acceptability of HIVST was defined as participants' general endorsement of HIVST as a testing mode, which may or may not include reasons why they preferred HIVST over another testing mode. If a study described a key population or group of participants that accepted HIVST as a mode of testing without describing why it was acceptable, it was excluded from our review.

\section{Willingness to Test}

We determined that willingness to test was closely related to acceptability but was future oriented. Many studies reported participants stated they would use HIVST in the future; however, if reasons why they would be willing to use HIVST were not described, the study was excluded.

\section{Likelihood to Test}

We defined likelihood to test as probability of using HIVST in the future. Several studies described likelihood of future use of HIVST over another method of testing. If reasons increasing their likelihood of using HIVST were not explicitly stated, articles were excluded from our review. 


\section{Preferences}

Preferences were defined as participants' values related to HIVST. This includes reasons why participants like or dislike HIVST, preferences about different HIVST options, or preferences related to using HIVST versus another testing mode. To investigate patient preferences regarding HIVST, we excluded all articles with that focused only on acceptability, willingness to test, and likelihood to test via HIVST that did not include preferences.

\section{Results}

The initial search yielded 686 records (Figure 1. PRISMA Diagram) and 286 additional records were identified via HIVST.org. The complete list of 972 records was crosschecked for duplicates, which were then removed from the list. The remaining 700 unique records were screened for eligibility through abstract review. A total of 561 abstracts were screened out and the remaining 139 articles underwent full-text review for eligibility. Of the 139 articles, 120 were excluded for lack of specificity regarding patient preferences for HIVST, or because they speculated about what participants might like about HIVST without directly capturing patient values. Thus, the scoping review yielded 19 articles with main findings that included HIVST patient preference in some form.

\section{Study Characteristics}

An overview of the characteristics of the 19 articles included is shown in Figure 2 (Results). Studies comprised a range of methods including surveys, focus groups, qualitative interviews, mixed methods, and one randomized controlled trial. Eleven studies used only adults as participants. The primary population studied was MSM, though other key populations included transgender individuals, sex workers, Black and Latino MSM, and people who inject drugs (PWID).

\section{Comparing HIVST to Clinic/ Community-Based Testing}

Many studies explored preference for HIVST compared with other testing modes. ${ }^{54-63}$ Huang et al (2016) provided

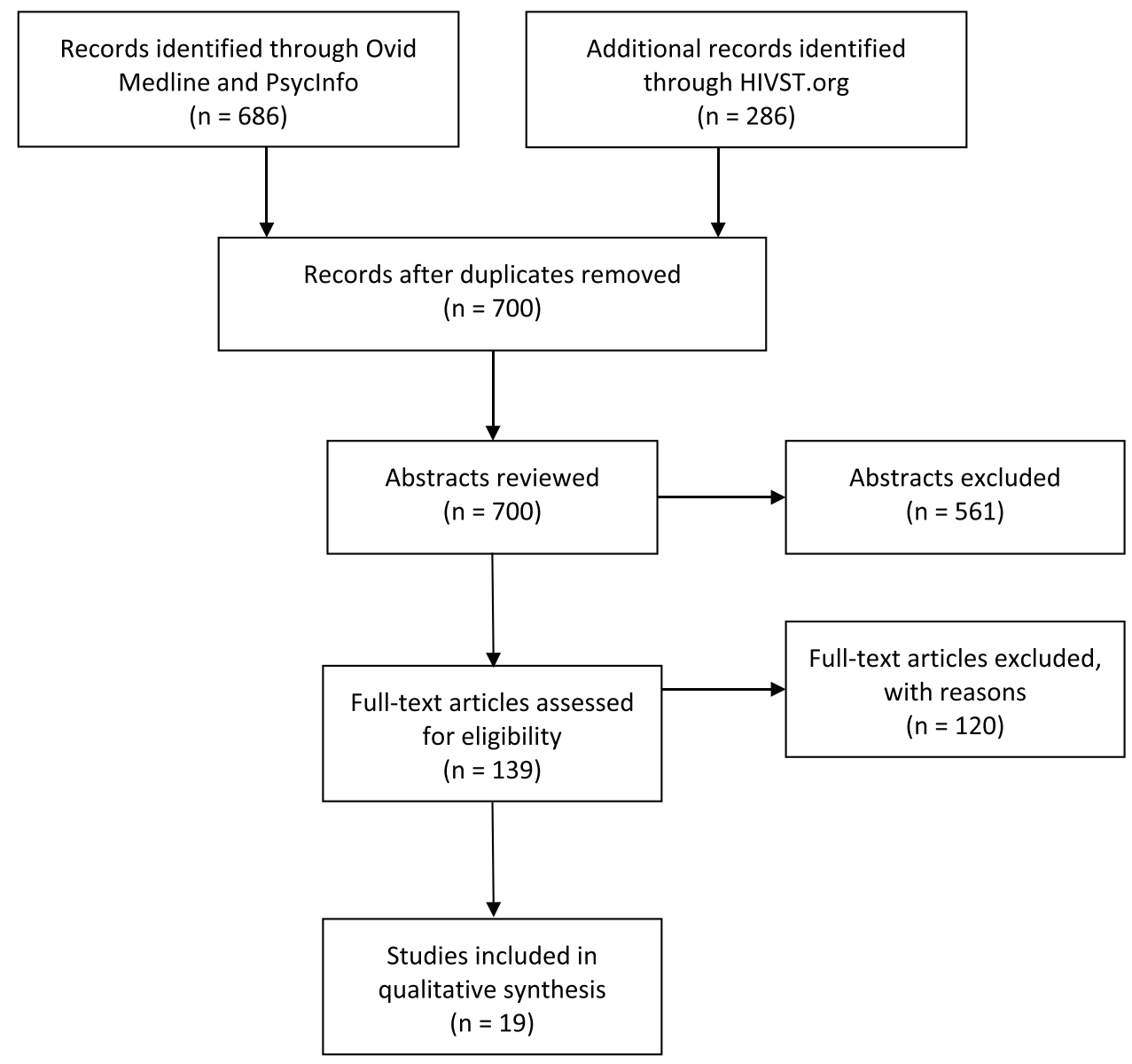

Figure I PRISMA Diagram. 


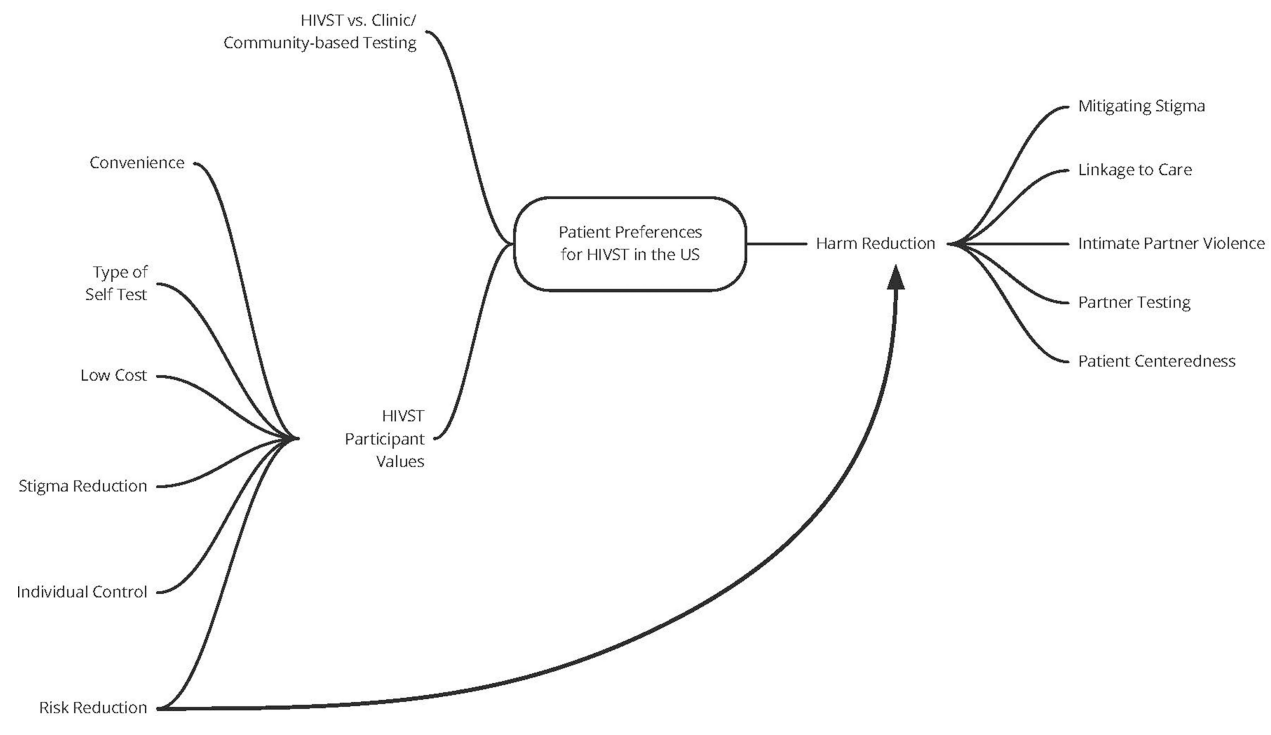

Figure 2 Results.

HIVST via mail to 122 Black and Latino MSM, and participants completed online surveys following completion of HIVST. Among participants who completed the survey, $68.4 \%$ preferred HIVST compared to other testing modes in the future. Similarly, Lippman et al (2016) found that $68 \%$ of participants in their study also preferred HIVST compared to clinic-based testing. Among Hubbard et al's (2020) sample in an HIVST pilot distribution study, $92 \%$ preferred HIVST to testing in a clinic. In a 2016 study of Black and Latino MSM who completed surveys before and after HIVST home delivery, 77\% of respondents preferred or somewhat preferred HIVST compared to traditional clinic-based testing. ${ }^{57}$ However, Freeman et al (2018) found that many participants saw HIVST mainly as an option to supplement but not replace their regular testing in clinics and overall preferred facility-based testing, and another study found social mediausing YMSM did not prefer HIVST over other testing options despite being generally favorable toward HIVST. ${ }^{62}$

\section{HIVST Participant Values}

As shown in Figure 2 (Results), we discovered six themes explaining what participants valued about HIVST and why they were willing to use this form of testing: convenience, types of tests (oral versus fingerstick), cost, stigma reduction, risk reduction, and self-control.

\section{Convenience}

The convenience of HIVST compared to testing in facilities was a value described by participants of many studies included in this review. ${ }^{55,56,58-70}$ Through focus groups and qualitative interviews of MSM, Freeman et al (2018) found that the convenience of purchasing an HIV test and having the test available when needed was valuable to participants. Hubbard et al (2020) also reported that 52\% of participants in their study liked that using HIVST allowed them to test at their own convenience. Participants in some studies also highlighted the value of avoiding repeat prevention counseling and other interactions with the healthcare system. ${ }^{55,61,68}$ Timeliness of test results was cited by participants in some studies as a reason for liking HIVST or recommending HIVST to others. $^{55,59}$ One 2014 study of Black and Latino MSM found that participants valued the convenience of choosing the location in which they would use the HIVST, as well the location in which they would receive the results. ${ }^{67}$

Similarly, the ease of use and/or simplicity of HIVST is valued by participants in several studies ${ }^{54-57,61,63,67,68}$ including one study with high-risk young adults who reported initial concerns about ability to use the test accurately, which were allayed once they completed a selftest. $^{68}$ Ease of use was associated with reports of convenience and higher acceptability. ${ }^{54}$ Following interviews with Black and Latino MSM, Young (2014) reported a positive consensus around the simplicity of HIVST that allowed individuals to perform the test without interrupting their schedule unlike clinic-based testing. In the second phase of their pilot distribution study, Hubbard et al (2020) found that $50 \%$ of those who 
reported using HIVST cited ease of use as a key reason for using this method.

\section{Types of Tests}

Several articles assessed participants' preferences for oral swab versus blood-based HIVST rapid tests among MSM. ${ }^{60-62,71}$ These studies found mixed results when assessing preferences for oral swab versus fingerstick selftests should that form of testing become available for at-home rapid use. One study found participants favored oral-swab testing, but that reluctance to use blood-based HIVST was reduced if tests for other sexually transmitted infection could be included in the test. ${ }^{71}$ Merchant et al (2017) found that young MSM would prefer blood-based HIVST as it was perceived to be accurate, quick, private, and convenient. While Schnall et al (2016) found most young adults in their study preferred blood-free testing overall, ${ }^{68}$ Frye et al (2018) found some participants had a strong preference for blood-based HIVST due to greater accuracy of test results and fewer false negatives compared to oral swab HIVST. ${ }^{70}$ Similarly, some studies found concerns regarding accuracy of HIVST results as compared to clinic-based testing. ${ }^{58,59,61,64,65,68,72}$

\section{Cost}

Cost is undoubtably the most frequent barrier to HIVST identified by participants of included studies. ${ }^{56,58,59,61,62,65,69,70,72}$ Brown et al (2016) found that the high cost of HIVST was a barrier to use and that if cost could be lowered participants would be more likely to use this method of testing. Similarly, Lippman et al (2016) found that participants identified cost as a "zero-sum factor," meaning if the cost of HIVST was prohibitive, participants would not utilize HIVST regardless of other preferences.

\section{Stigma Reduction}

The reduction of HIV and STI stigma was important to many participants. ${ }^{56,59,61,62,64-66,68-70}$ For many, especially sexual and gender minorities, performing an HIV test at home rather than at a clinic or another medical facility allowed them reprieve from the stigma and discrimination they often experience while attempting to access health care services. ${ }^{65,68}$ Several articles highlighted the value of HIVST in reducing anxiety and stigma related to testing. ${ }^{2,9,65,67,69}$ Using HIV along with patient-delivered partner therapy (PDPT) was identified as a value; participants reported that using HIVST in PDPT helped them avoid the stigma of an STI clinic and empowered them in their control of their own health. ${ }^{64}$ Furthermore, the peace of mind related to knowing the partners' status was an important value of HIVST. ${ }^{64}$ There were some, however, that expressed anxiety related to purchasing a self-test, specifying the potential risk of being seen purchasing the test or having someone find it in their home, suggesting that access to HIVST is not without stigma. ${ }^{61,66,69,70}$

\section{Risk Reduction}

The potential to use HIVST along with partners was identified as a benefit by participants in several studies. ${ }^{2,6,58,64,65,72}$ Lippman et al (2016) found transgender women were interested in HIVST for testing partners and to inform sexual decision-making. ${ }^{56}$ John (2018) reported that some participants said that including HIVST in PDPT could create earlier opportunities to identify HIV-positive partners; however, others feared introducing HIVST to their partners would be poorly received and possibly even increase risk of violence, especially in the case of a positive result. ${ }^{58,64}$ One study found that participants reported using HIVST as a prevention tool that allowed them more clarity when deciding whether to have condomless sex. ${ }^{65}$ Giguere et al (2016) conducted a feasibility study of HIVST with including cisgender male and transfeminine sex workers. Sixty-eight percent of participants reported being likely to use HIVST to test clients prior to sex work and also reported that uptake of HIVST may increase if tests are made available over the counter. However, some participants identified potential danger in the use of HIVST either with sex work clients or in front of them to demonstrate they were HIV negative. ${ }^{72}$

\section{Individual Control}

The ability for one to control how, when, and for what reasons to take a self-test is a theme that intersects with nearly all of those previously discussed. The ability to test more often or as soon as possible was important for some. ${ }^{55,58,62,64,70}$ HIVST allowed participants to control when and where to use HIV testing in their lives without having to interact with clinics or community-based organizations (CBOs). The value of individual control over HIV testing was seen as a way to alleviate testing anxiety and stigma. ${ }^{67,69}$ Control over how results are used has been seen both at the individual and dyadic levels as a decision-making risk-reduction strategy. For transgender individuals, HIVST provides them the ability to test themselves, especially in instances where they do not hold the power in their social and sexual relationships. ${ }^{56}$ Selfcontrol is also connected with increased perceived 
privacy. ${ }^{55,56,58,59,61-63,65-69}$ Privacy often extends to the idea of confidentiality, highlighted in several articles. $^{59,64,66,67,69}$ Furthermore, individuals felt empowered by HIVST because it gave them control over their health as well as their partners' health. ${ }^{6,64}$

\section{Linkage to Care}

Testing outside of traditional HIV services systems was seen as a barrier to HIVST for many participants, including some studies where participants expressed concerns about gaining immediate support and linkage to services if individual tests were received alone at home. ${ }^{56,58,59,61,69}$ One study observed that some participants preferred to test in a clinic if they thought they might be positive, although $64 \%$ of participants preferred to read test results alone. ${ }^{56}$ Similarly, Cushman et al (2019) found that most MSM preferred HIVST to traditional clinic HIV testing but that while they preferred to receive negative results via website, text, or email, they want to receive positive results from a provider via phone or in person. ${ }^{60}$ However, more self-control over testing, for some, meant being able to test more often which provides the opportunity for earlier detection and care initiation. ${ }^{61,66}$ Explicit access to postHIVST counseling, support, and direct linkage to care was seen as essential for HIVST to work. $^{66}$

\section{Discussion}

We found 19 articles published between January 2014April 2020 that included findings specifying HIVST preferences. Overall, HIVST was preferred to more traditional testing (eg, clinic or $\mathrm{CBO}$ ). Six primary factors emerged as important to preferring HIVST. 1) Convenience: where the test could be obtained/taken, ease of testing, and timeliness of results. 2) Type of test: oral swab was mostly preferred over blood-based tests; however, there was some concern about the specificity of non-blood-based tests. 3) Risk reduction: using HIVST as a tool for risk-reduction decision-making and engaging with partners. 4) Cost: an important barrier to being able to access HIVST was affordability. 5) Stigma reduction: HIVST was seen as an opportunity to increase privacy and reduce HIV stigma associated with in-person traditional HIV testing. 6) Selfcontrol: HIVST provided more opportunity for self-control over how, when, and for what reasons to take and use results from an HIVST.

There are several limitations to consider when interpreting our findings. First, we only used two databases to search the literature and therefore missed any articles not included therein. However, these two databases are the primary sources for medical and public health literature. Additionally, we reviewed citations of systematic or scoping reviews as well as meta-analyses and searched an additional HIVST-related database, thereby increasing the likelihood that our search strategies capture the full breadth of research. We also only included published manuscripts. By not including conference abstracts and grey literature, we may have missed some more recent studies that have not yet been published. Nonetheless, we believe we reached saturation with our methods given we repeatedly came across the same set of studies in our searches and in their references to each other. Limiting our inclusion criteria to the US provides only a partial picture of current practices in HIVST. Because many studies on HIVST have taken place in global contexts, we intentionally focused this review domestically, where there has been less research on this topic and where HIVST has been available to individuals beyond research studies. Additionally, our focus is on patient preferences, which differ by cultural settings, further informing our decision to limit to one geographic region. The final reason we wanted to narrow our focus to the US was because our findings may have US research and policy implications.

\section{HIVST as a Tool for Harm Reduction}

Considering these findings, much of what makes HIVST attractive to individuals is their ability to self-determine how HIVST can be best integrated into their lives as a harm reduction tool for stigma and sexual risk mitigation. For many, especially sexual and gender minorities, the possibility of testing for HIV at home rather than at a clinic or HIV-focused $\mathrm{CBO}$ allows them reprieve from the stigma and discrimination often experienced while attempting to access health care services. The privacy of HIVST may also help increase confidentiality and decrease opportunities for "outing" and other harmful exposures. HIVST was seen as a tool that helps individuals to make informed sexual decisions more freely. Individuals felt HIVST gave them control over their own health and empowered them to make decisions that are best for their own and their partners' sexual health. This ability to have self-control of testing was especially important in instances where individuals do not hold the power in their social and sexual relationships, thereby potentially reducing potential harm within dyadic relationships.

Promoting HIVST as an important tool for sexual risk harm reduction approaches in the context of biobehavioral 
HIV prevention is largely understudied and underutilized in applied settings. Despite findings that HIVST improves individual control and reduces stigma, the decision to choose this mode of testing was complicated by the need to balance privacy with support. ${ }^{56}$ This may be particularly true for those with low social resources and high fear of stigma. Reducing the harms of stigma through HIVST has the potential to promote increased and regular testing among high-risk populations that are currently missed in many prevention efforts. Incorporating HIVST as a tool for harm reduction in HIV prevention messaging has the potential to empower individuals to test more frequently and use current results in sexual decision-making. Connecting HIVST to prevention and treatment services (eg, PrEP, TasP) has the potential to increase effectiveness of both primary and secondary HIV prevention efforts. For this to be effective, the cost will have to be driven down as this is still a barrier for many oppressed populations.

Findings from these studies largely focused on MSM. Fewer data were included on transgender populations, sex workers, and people who inject drugs. Other understudied populations include heterosexual populations; unhoused populations; Black, Indigenous, and other women of color (BIWOC), transgender men, and MSM of color who are not Black or Latinx. While HIVST has not been rigorously studied in all communities as risk for HIV, there is substantial evidence to suggest that HIVST is feasible for use with many oppressed and other populations, and there are aspects of HIVST that are beneficial and preferred over traditional testing approaches.

Further implementation studies that rigorously test how best to scale up HIVST in community settings are needed. One recent study addressed this issue and recommended that HIVST should be incorporated into community-based testing programs as a supplement but not a replacement to traditional testing models in order to reach those who might not otherwise be tested. The authors also noted that more over-the-counter options for HIV self-testing would enhance competitive pricing and make HIVST more affordable, a barrier that was reported in many of the studies included in our review. ${ }^{73}$ Other HIVST innovative delivery models have been presented in conference abstracts, although not all have appeared in peer-reviewed literature. The presence of these abstracts is encouraging because it suggests that findings from these studies will be available for researchers and providers to build on, expanding the reach and availability of HIVST services and research. Overall, the breadth of research addressing
HIVST services indicates we may be on the cusp of many important new directions for HIVST in applied settings.

Additional studies are needed to document how to overcome known barriers (eg, cost) and to identify unknown barriers beyond the research setting. Future studies should also explore how to integrate HIVST into prevention messaging, improve ongoing access, ensure linkages to treatment and prevention care, and better understand how individuals use HIVST in applied settings. Given the potential for HIVST to decrease stigma and decrease harm for key populations with unique values, needs, and barriers, there is an ideal opportunity for academic-community partnerships to bring resources to communities while rigorously evaluating what works.

\section{Conclusion}

Although HIVST has its limitations, our findings regarding patient preferences for this method of testing suggest that it may meet the needs of key populations, reduce stigma, aid in sexual decision-making, and potentially increase regular testing among those currently missed in prevention messaging. Evidence suggests that HIVST is feasible, has high acceptability, and is valued by members of several key populations for its convenience, ease of swab testing, use in risk reduction, opportunities for stigma reduction, possibility of self-control, and linkage to care. As a means of empowering individuals to engage with testing on their own terms while providing pathways to prevention and care support, HIVST has the potential to be a powerful harm reduction tool in the HIV biobehavioral prevention toolkit.

\section{Disclosure}

The authors report no conflicts of interest in this work.

\section{References}

1. Centers for Disease Control and Prevention. Estimated HIV incidence and prevalence in the United States, 2010-2015. 2018. Available from: http://www.cdc.gov/hiv/library/reports/hiv-surveillance.html.

Accessed September 18, 2020.

2. Wolf RC, Bingham T, Millett G, Wilcher R. Building the evidence base to optimize the impact of key population programming across the HIV cascade. J Int AIDS Soc. 2018;21(Suppl):5). doi:10.1002/ jia2.25146

3. Okeke N, McFarland W, Raymond H. Closing the gap? The HIV continuum in care for African-American men who have sex with men, San Francisco, 2004-2014. AIDS Behav. 2017;21(6):17 41-1744. doi:10.1007/s10461-016-1472-0

4. Chandler CJ, Sang JM, Bukowski LA, et al. Characterizing the HIV care continuum among a community sample of black men who have sex with men in the United States. AIDS Care. 2019;31(7):816-820. doi:10.1080/09540121.2018.1549724 
5. Risher K, Mayer K, Beyrer C. The HIV treatment cascade in men who have sex with men, people who inject drugs and sex workers. Curr Opin HIV AIDS. 2015;10(6):420. doi:10.1097/COH.0000000 000000200

6. UNAIDS. Ending AIDS: Progress Towards the 90-90-90targets.

7. Staveteig S, Croft TN, Kampa KT, Head SK. Reaching the 'first 90': gaps in coverage of HIV testing among people living with HIV in 16 African countries. PLoS One. 2017;12(10):e0186316. doi:10.1371/ journal.pone. 0186316

8. Kuehn B. Gaps in HIV testing for black men. JAMA. 2018;320 (10):966.

9. DiNenno EA, Prejean J, Irwin K, et al. Recommendations for HIV screening of gay, bisexual, and other men who have sex with menUnited States, 2017. MMWR Morb Mortal Wkly Rep. 2017;66 (31):830. doi:10.15585/mmwr.mm6631a3

10. Skarbinski J, Rosenberg E, Paz-Bailey G, et al. Human immunodeficiency virus transmission at each step of the care continuum in the United States. JAMA Intern Med. 2015;175(4):588-596. doi:10.1001/ jamainternmed.2014.8180

11. Facts about in-home HIV testing. US Food and Drug Administration. 2020. Available from: https://www.fda.gov/consumers/consumerupdates/facts-about-home-hiv-testing. Accessed September 25, 2020

12. US Food and Drug Administration. Information regarding the Home Access HIV-1 Test System. Available from: https://www.fda.gov/vac cines-blood-biologics/approved-blood-products/information-regardinghome-access-hiv-1-test-system. Accessed September 25, 2020

13. Tucker JD, Bien CH, Peeling RW. Point-of-care testing for sexually transmitted infections: recent advances and implications for disease control. Research Support, N.I.H., Extramural Research Support, Non-U.S. Gov't Review. Curr Opin Infect Dis. 2013;26(1):73-79. doi:10.1097/QCO.0b013e32835c21b0

14. Pant Pai N, Sharma J, Shivkumar S, et al. Supervised and unsupervised self-testing for HIV in high- and low-risk populations: a systematic review. Research Support, Non-U.S. Gov't review systematic review. PLoS Med Publ Lib Sci. 2013;10(4):e1001414.

15. Gaydos CA, Hsieh YH, Harvey L, et al. Will patients "opt in" to perform their own rapid HIV test in the emergency department? Research Support, N.I.H., Extramural Research Support, Non-U.S. Gov't. Ann Emerg Med. 2011;58(1 Suppl 1):S74-8. doi:10.1016/j. annemergmed.2011.03.029

16. Gaydos CA, Solis M, Hsieh Y-H, Jett-Goheen M, Nour S, Rothman RE. Use of tablet-based kiosks in the emergency department to guide patient HIV self-testing with a point-of-care oral fluid test. Int J STD AIDS. 2013;24(9):716-721. doi:10.1177/09564624 13487321

17. Marlin RW, Young SD, Bristow CC, et al. Piloting an HIV self-test kit voucher program to raise serostatus awareness of high-risk African Americans, Los Angeles. Research Support, N.I.H., Extramural. BMC Public Health. 2014;14:1226. doi:10.1186/14712458-14-1226

18. Chang W, Matambanadzo P, Takaruza A, et al. Effect of Prices, Distribution Strategies, and Marketing on Demand for HIV Self-testing in Zimbabwe: a Randomized Clinical Trial. JAMA Net Open. 2019;2(8):e199818. doi:10.1001/jamanetworkopen.2019.9818

19. Meyerson BE, Emetu RE, Sanders SA, Bailey MM, Ryder PT, Armstrong J. Preferences of gay and bisexual men for pharmacy-based HIV testing and over-the-counter HIV tests. Lgbt Health. 2014;1(3):225-228. doi:10.1089/lgbt.2014.0010

20. Fernández-Balbuena S, de la Fuente L, Hoyos J, et al. Highly visible street-based HIV rapid testing: is it an attractive option for a previously untested population? A cross-sectional study. Sex Transm Infect. 2014;90(2):112-118. doi:10.1136/sextrans-2013051234

21. Ko JS, Stafylis C, Klausner JD. Mobile health promotion of human immunodeficiency virus self-testing in the United States. mHealth. 2020;6:10. doi:10.21037/mhealth.2019.10.05
22. Brown AN, Djimeu EW, Cameron DB. A review of the evidence of harm from self-tests. AIDS Behav. 2014;18(Suppl 4):S445-9. doi:10.1007/s10461-014-0831-y

23. Figueroa C, Johnson C, Ford N, et al. Reliability of HIV rapid diagnostic tests for self-testing compared with testing by health-care workers: a systematic review and meta-analysis. Comparative study meta-analysis research support, Non-U.S. Gov't systematic review. Lancet HIV. 2018;5(6):e277-e290. doi:10.1016/S2352-3018(18)30 044-4

24. Krause J, Subklew-Sehume F, Kenyon C, Colebunders R. Acceptability of HIV self-testing: a systematic literature review. Review Systematic Review. BMC Public Health. 2013;13(735).

25. Stevens DR, Vrana CJ, Dlin RE, Korte JE, Global A. Review of HIV Self-testing: themes and Implications. Review. AIDS Behav. 2018;22 (2):497-512.

26. Johnson CC, Kennedy C, Fonner V, et al. Examining the effects of HIV self-testing compared to standard HIV testing services: a systematic review and meta-analysis. Meta-Analysis Review Systematic Review. J Int AIDS Soc. 2017;20(1):21594. doi:10.7448/ IAS.20.1.21594

27. Adebayo OW, Salerno JP. Facilitators, barriers, and outcomes of self-initiated HIV testing: an integrative literature Review. Res Theory Nurs Pract. 2019;33(3):275-291. doi:10.1891/1541-6577.33. 3.275

28. Chakroun M, Razik F, Karkouri M, Fall Malick Z, Benothman H, George Hermez J. HIV epidemic in the Maghreb. Magnitude, trend and management. Review. Tunisie Medicale. 2018;96(10-11): 599-605.

29. Chikwari CD, Dringus S, Ferrand RA. Barriers to, and emerging strategies for, HIV testing among adolescents in sub-Saharan Africa. Research Support, Non-U.S. Gov't Review. Curr Opin HIV AIDS. 2018;13(3):257-264. doi:10.1097/COH.00000000000 00452

30. Hamilton A, Shin S, Taggart T, et al. HIV testing barriers and intervention strategies among men, transgender women, female sex workers and incarcerated persons in the Caribbean: a systematic review. Review. Sex Transm Infect. 2019;10:10.

31. Zhang C, Li X, Brecht ML, Koniak-Griffin D. Can self-testing increase HIV testing among men who have sex with men: A systematic review and meta-analysis. Meta-Analysis Systematic Review. PLoS One. 2017;12(11):e0188890.

32. Clark HA, Oraka E, DiNenno EA, et al. Men who have Sex with Men (MSM) Who have not previously tested for HIV: results from the MSM Testing Initiative, United States (2012-2015). AIDS Behav. 2019;23(2):359-365. doi:10.1007/s10461-018-2266-3

33. Guidelines on HIV Self-Testing and Partner Notification: Supplement to Consolidated Guidelines on HIV Testing Services. 2016;92 41549866.

34. Qin Y, Han L, Babbitt A, et al. Experiences using and organizing HIV self-testing. Research Support, N.I.H., Extramural Research Support, Non-U.S. Gov't Systematic Review. AIDS. 2018;32 (3):371-381.

35. Campbell CK, Lippman SA, Moss N, Lightfoot M. Strategies to increase HIV testing among MSM: a synthesis of the literature. Systematic review. AIDS Behav. 2018;22(8):2387-2412. doi:10.10 07/s10461-018-2083-8

36. Cooke IJ, Jeremiah RD, Moore NJ, et al. Barriers and facilitators toward HIV testing and health perceptions among African-American men who have sex with women at a South Side Chicago Community Health Center: a pilot study. Front Public Health. 2017;4:286. doi:10.3389/fpubh.2016.00286

37. Gamarel KE, Nelson KM, Stephenson R, et al. Anticipated HIV stigma and delays in regular HIV testing behaviors among sexually-active young gay, bisexual, and other men who have sex with men and transgender women. AIDS Behav. 2018;22(2):522-530. doi:10.1007/s10461-017-2005-1 
38. Smit PJ, Brady M, Carter M, et al. HIV-related stigma within communities of gay men: a literature review. AIDS Care. 2012;24 (4):405-412. doi:10.1080/09540121.2011.613910

39. Golub SA, Gamarel KE. The impact of anticipated HIV stigma on delays in HIV testing behaviors: findings from a community-based sample of men who have sex with men and transgender women in New York City. AIDS Patient Care STDS. 2013;27(11):621-627. doi:10.1089/apc.2013.0245

40. Arnold EA, Rebchook GM, Kegeles SM. 'Triply cursed': racism, homophobia and HIV-related stigma are barriers to regular HIV testing, treatment adherence and disclosure among young Black gay men. Cult Health Sex. 2014;16(6):710-722. doi:10.1080/136910 58.2014.905706

41. Wise JM, Ott C, Azuero A, et al. Barriers to HIV testing: patient and provider perspectives in the deep south. AIDS Behav. 2019;23 (4):1062-1072. doi:10.1007/s10461-018-02385-5

42. Bukowski LA, Chandler CJ, Creasy SL, Matthews DD, Friedman MR, Stall RD. Characterizing the HIV care continuum and identifying barriers and facilitators to HIV diagnosis and viral suppression among black transgender women in the United States. J Acquir Immune Def Syndr. 2018;79(4):413. doi:10.1097/QAI.0000 000000001831

43. Shannon K, Crago A-L, Baral SD, et al. The global response and unmet actions for HIV and sex workers. The Lancet. 2018;392 (10148):698-710. doi:10.1016/S0140-6736(18)31439-9

44. King EJ, Maman S, Bowling JM, Moracco KE, Dudina V. The influence of stigma and discrimination on female sex workers' access to HIV services in St. Petersburg, Russia. AIDS Behav. 2013;17 (8):2597-2603. doi:10.1007/s10461-013-0447-7

45. Derlega VJ, Winstead BA, Brockington JE Jr. AIDS stigma among inmates and staff in a USA state prison. Int J STD AIDS. 2008;19 (4):259-263. doi:10.1258/ijsa.2007.007141

46. Andrinopoulos K, Kerrigan D, Figueroa JP, Reese R, Ellen JM. HIV coping self-efficacy: a key to understanding stigma and HIV test acceptance among incarcerated men in Jamaica. AIDS Care. 2010;22(3):339-347. doi:10.1080/09540120903193633

47. Yan Z-H, Lin J, Xiao W-J, et al. Identity, stigma, and HIV risk among transgender women: a qualitative study in Jiangsu Province, China. Infect Dis Poverty. 2019;8(1):1-9. doi:10.1186/s40249-0190606-9

48. Sharma A, Chavez PR, MacGowan RJ, et al. Willingness to distribute free rapid home HIV test kits and to test with social or sexual network associates among men who have sex with men in the United States. AIDS Care. 2017;29(12):1499-1503. doi:10.1080/ 09540121.2017.1313386

49. Rael CT, Giguere R, Lopez-Rios J, et al. Transgender women's experiences using a home HIV-testing kit for partner-testing. AIDS Behav. 2020;19:19.

50. Giguere R, Lopez-Rios J, Frasca T, et al. Use of HIV self-testing kits to screen clients among transgender female sex workers in New York and Puerto Rico. AIDS Behav. 2020;24(2):506-515. doi:10.1007/ s10461-019-02730-2

51. Figueroa C, Johnson C, Verster A, Baggaley R. Attitudes and acceptability on HIV self-testing among key populations: a literature review. Review. AIDS Behav. 2015;19(11):1949-1965. doi:10.1007/ s10461-015-1097-8

52. Marlatt GA. Harm reduction: come as you are. Addict Behav. 1996;21(6):779-788. doi:10.1016/0306-4603(96)00042-1

53. Hawk M, Coulter R, Egan J, Friedman M, Tula M, Kinsky S. Harm reduction principles for healthcare settings. Harm Reduct J. 2017;14:70. doi:10.1186/s12954-017-0196-4

54. Huang E, Marlin RW, Young SD, Medline A, Klausner JD. Using Grindr, a smartphone social-networking application, to increase HIV self-testing among black and latino men who have sex with men in Los Angeles, 2014. AIDS Educ Prev. 2016;28(4):341-350. doi:10.15 21/aeap.2016.28.4.341
55. Hubbard SJ, Ma M, Wahnich A, Clarke A, Myers JE, Saleh LD. \#testathome: implementing two phases of an HIV self-testing program through CBO partnerships in New York City. Sex Transm Dis. 2020;05:05.

56. Lippman SA, Moran L, Sevelius J, et al. Acceptability and Feasibility of HIV self-testing among transgender women in San Francisco: a mixed methods pilot study. Research Support, N.I.H., Extramural Research Support, Non-U.S. Gov't. AIDS Behav. 2016;20(4): 928-938. doi:10.1007/s10461-015-1236-2

57. Rosengren AL, Huang E, Daniels J, Young SD, Marlin RW, Klausner JD. Feasibility of using Grindr(TM) to distribute HIV self-test kits to men who have sex with men in Los Angeles, California. Sex Health. 2016;23:23.

58. Brown W III, Carballo-Dieguez A, John RM, Schnall R. Information, motivation, and behavioral skills of high-risk young adults to use the HIV self-test. AIDS Behav. 2016;20(9):2000-2009. doi:10.1007/ s10461-016-1309-x

59. Catania JA, Dolcini MM, Harper GW, et al. Bridging barriers to clinic-based HIV testing with new technology: translating self-implemented testing for African American youth. Transl Behav Med. 2015;5(4):372-383. doi:10.1007/s13142-015-0331-2

60. Cushman TA, Graves SK, Little SJ. Attitudes and preferences regarding the use of rapid self-testing for sexually transmitted infections and HIV in San Diego area men who have sex with men. Open Forum Infect Dis. 2019;6(3):ofz043. doi:10.1093/ofid/ ofz043

61. Freeman AE, Sullivan P, Higa D, et al. Perceptions of HIV self-testing among men who have sex with men in the United States: a qualitative analysis. AIDS Educ . 2018; 30(1):47

62. Merchant RC, Clark MA, Liu T, et al. Preferences for oral fluid rapid HIV self-testing among social media-using young black, Hispanic, and white men-who-have-sex-with-men (YMSM): implications for future interventions. Public Health. 2017;145:7-19.

63. Merchant RC, Clark MA, Liu T, et al. Comparison of Home-Based Oral Fluid Rapid HIV Self-Testing Versus Mail-in Blood Sample Collection or Medical/Community HIV Testing By Young Adult Black, Hispanic, and White MSM: results from a Randomized Trial. Randomized Controlled Trial Research Support, N.I.H., Extramural. AIDS Behav. 2018;22(1):337-346.

64. John SA. Addressing missed opportunities for HIV testing by including rapid-HIV self-testing kits with patient-delivered partner therapy. Sexual Res Soc Policy. 2018;15(4):387-397. doi:10.1007/s13178017-0316-7

65. Lippman SA, Koester KA, Amico KR, et al. Client and provider perspectives on new HIV prevention tools for MSM in the Americas. Multicenter Study Research Support, N.I.H., Extramural. PLoS One. 2015;10(3):e0121044. doi:10.1371/journal. pone. 0121044

66. Medline A, Daniels J, Marlin R, et al. HIV testing preferences among MSM members of an LGBT community organization in Los Angeles. J Assoc Nurs AIDS Care. 2017;28(3):363-371. doi:10.1016/j.jana.20 17.01.001

67. Young SD, Daniels J, Chiu CJ, et al. Acceptability of using electronic vending machines to deliver oral rapid HIV self-testing kits: a qualitative study. Research Support, N.I.H., Extramural Research Support, Non-U.S. Gov't. PLoS One. 2014;9(7):e103790. doi:10.13 71/journal.pone. 0103790

68. Schnall R, John RM, Carballo-Dieguez A. Do high-risk young adults use the HIV self-test appropriately? Observations from a Think-Aloud Study. Observational Study Research Support, N.I.H., Extramural. AIDS Behav. 2016;20(4):939-948. doi:10.1007/s10461015-1240-6

69. Frye V, Wilton L, Hirshfied S, et al. "Just because it's out there, people aren't going to use it." HIV self-testing among young, Black MSM, and transgender women. AIDS Patient Care STDS. 2015;29 (11):617-624. doi:10.1089/apc.2015.0100 
70. Frye V, Wilton L, Hirshfield S, et al. Preferences for HIV test characteristics among young, Black Men Who Have Sex With Men (MSM) and transgender women: implications for consistent HIV testing. Research Support, N.I.H., Extramural. PLoS One. 2018;13 (2):e0192936. doi:10.1371/journal.pone.0192936

71. Balan I, Frasca T, Ibitoye M, Dolezal C, Carballo-Dieguez A. Fingerprick versus oral swab: acceptability of blood-based testing increases if other STIs can be detected. comparative study. AIDS Behav. 2017;21(2):501-504. doi:10.1007/s10461-016-1497-4

72. Giguere R, Frasca T, Dolezal C, et al. Acceptability of three novel HIV prevention methods among young male and transgender female sex workers in Puerto Rico. AIDS Behav. 2016;20(10):2192-2202. doi:10.1007/s10461-016-1387-9
73. Steehler K, Siegler AJ. Bringing HIV self-testing to scale in the United States: a review of challenges, potential solutions, and future opportunities. J Clin Microbiol. 2019;57(11):e00257-19. doi:10.11 28/JCM.00257-19

74. Niederstadt C, Droste S. Reporting and presenting information retrieval processes: the need for optimizing common practice in health technology assessment. Int J Technol Assess Health Care. 2010;26 (4):450-457. doi:10.1017/S0266462310001066

\section{Publish your work in this journal}

Patient Preference and Adherence is an international, peer-reviewed, open access journal that focusing on the growing importance of patient preference and adherence throughout the therapeutic continuum. Patient satisfaction, acceptability, quality of life, compliance, persistence and their role in developing new therapeutic modalities and compounds to optimize clinical outcomes for existing disease states are major areas of interest for the journal. This journal has been accepted for indexing on PubMed Central. The manuscript management system is completely online and includes a very quick and fair peer-review system, which is all easy to use. Visit http:// www.dovepress.com/testimonials.php to read real quotes from published authors. 